\title{
Disease of consumption to extensively drug resistance tuberculosis: Race of techniques to enhance diagnosis
}

\author{
Jureka Mankotia ${ }^{\mathrm{a}}$, Akant Kaushal ${ }^{\mathrm{b}}$, Azhar Khan ${ }^{\mathrm{c}^{*}}$ \\ ${ }^{a}$ Department of Applied Sciences and Biotechnology, Shoolini University, Solan, Himachal Pradesh \\ ${ }^{b}$ TB Sanatorium, Dharampur Solan, Himachal Pradesh \\ 'Molecular and Immuno-Parasitology Research Laboratory, Faculty of Applied Sciences and Biotechnology, Shoolini University, Solan, \\ Himachal Pradesh
}

Received 7th May 2020 / Accepted 13th October 2020

\begin{abstract}
Tuberculosis is one of the most ancient infectious diseases known to mankind. Detection of the causative organism, Mycobacterium tuberculosis, has experienced a series of advances from the late 19th century through early 21 st century. The hierarchy of diagnosis ranges from smear microscopy with traditional Ziehl-Neelsen staining to advanced light-emitting diode-fluorescence microscopy staining to both solid and liquid culture, and more recently, to genotypic methods like nucleic acid amplification. Each procedure has its own merits and drawbacks in terms of specificity, sensitivity, minimum detectible number of bacilli, and most crucially, turnover time. The latest diagnostic tools, like geneXpert and line probe assays, have gone a step further by detecting drug resistance. Both early and accurate diagnosis based on the drug susceptibility test implies precise and prompt initiation of treatment, thereby curtailing not only the agony of patient but also the burden tuberculosis places on the health system.
\end{abstract}

Keywords: Mycobacterium tuberculosis, microscopy, culture, rifampicin, drug resistance

\section{INTRODUCTION}

Tuberculosis (TB) has existed for millennia and remains a major public health concern globally. The number of deaths caused by TB, which was known as the consumption, phthisis and white plague in the beginning of $20^{\text {th }}$ century is unclear, but seems likely to be high. Tuberculosis has been prevalent in India since at least 1500 BCE, when its symptoms of wounds, hunger and fatigue were noted (Barberis et al., 2017) Historically, the fatality rate of the disease has been very high, as there was no diagnostic or treatment modality available.

Despite many decades of development of both methods for diagnosis and treatment, TB remains one of the top 10 causes of deaths globally, ranking even above HIV/AIDS. Globally, there are around 10 million cases of tuberculosis and 1.2 million deaths have been reported. India ranks first in terms of absolute number of TB cases, contributing $27 \%$ of the global burden, while $35 \%$ of global deaths due to TB occur in that country( India TB report, 2019)

The diagnosis of TB was mostly symptombased until Robert Koch's 1882 discovery of Mycobacterium tuberculosis, its causative organism, and development of a technique for staining the bacilli(Barberis et al., 2017). After finding the TB bacteria, researchers were reluctant to grow and

*Author for correspondence: Dr. Azhar Khan, Associate Professor, Faculty of Applied Sciences and Biotechnology, Shoolini University, Solan. Email mk.azhar1@gmail.com 
study its characteristics. Although animal models were promising, at that time, direct study of the causative microbe required a pure tubercle bacilli population.(Herzog, 1998).This gave rise to a search for an appropriate medium to grow TB bacilli.

The first medium used to successfully grow the TB bacilli was a solid medium suggested by Dorset in 1902. In 1915, Petroff modified the medium by adding gentian violet, which provided a contrast to the tubercle colonies and the white medium.(WHO, 2009). He also introduced a sodium hydroxide treatment for decontamination and homogenization (Van Pinxteren et al., 2000).Eventually, Petroff's methods replaced all previous methods used to isolate tuberculosis. Even though the growth of TB bacilli remained slow and time-consuming, with maintenance of a pure and contamination-free culture quite challenging, Petroff's methods replaced the need to use guinea pigs as the primary means to isolate TB.

In the 1990's, Middlebrook introduced an advanced, liquid medium method of culture for research (Singh et al., 2019). Although all the diagnostic modalities were promising, there was a need of fast and accurate diagnosis. This led researchers to brainstorm and produce techniques for diagnosis at point of care, including nucleic acid amplification and real-time polymerase chain reaction (PCR) assays. In this review, we seek to offer an overview of past and presently evolving technologies in diagnosis of TB and its resistance to first- and second-line drugs. (WHO, 2009).

\section{Light smear microscopy}

Smear microscopy is a technique initially developed over a century ago after Koch's discovery of tubercle bacilli and iteratively refined since. Because it utilizes cheap equipment and materials, most developing countries uses sputum microscopy as the basic tool for diagnosis (Parsons et al., 2011). Microscopy is highly specific for M. tuberculosis; it appears curved, long and beaded; however, this method does require expertise, and its accuracy is observation dependent. The sensitivity of microscopy is $60 \%$ with a detection threshold $10^{4}-10^{5}$ bacilli $/ \mathrm{ml}$ under optimal conditions (Singhal et al., 2015) However, with an increase in testing burden; microscopy meets with technical and operational constraints.

Therefore, light microscopy using traditional Ziehl-Neelsen (ZN) staining for Acid fast bacilli $\mathrm{AFB}$ has been replaced by fluorescent staining in high-throughput setups. LED microscopes were recently approved by the World Health Organization (WHO) for countries dealing with a high burden of comorbid HIV and TB(Reza et al., 2013). Though the microscopy technique is very much dependent on the technical and operational subjects, it can be enhanced by modifying the factors influencing microscopy, like sample collection, staining techniques and microscope quality. As a manual process, it is subjected to human error (Chakravorty et al., 2005). Sample collection and site must be correct to get the best results from the technique. For example, to diagnose pulmonary tuberculosis, the collected sample must originate from the respiratory tract using induced sputum, Bronchial Alveolar Lavage (BAL) or a lung biopsy. The specimen required for extra-pulmonary $\mathrm{TB}$ is particularly site dependent. The most common sites of infection are biopsies, aspirates, pus, urine and sterile body fluids including cerebrospinal, synovial, pleural, pericardial or peritoneal fluids (Cuevas et al., 2011) However, the sensitivity of microscopy is a concern because of the paucibacillary nature of extra-pulmonary samples.

\section{Culture medium-based techniques}

The standardized procedures are conventional methods using Lowenstein-Jensen medium which took 8-12 weeks to grow $M$. tuberculosis. These methods were also used for drug susceptibility testing (DST) to test the laboratory strains for resistance. This technique is widely adapted as it was relatively inexpensive. But it requires a minimum 6-8-week period, which causes challenges of diagnosis and treatment due to the delay in results, increasing morbidity and mortality among patients.

A research media by Middlebrook in the form of liquid culture came as a boon to the field in the 1990 s decreasing testing turnaround times to $2-4$ weeks. The most common technique used a BACTEC460 (Tuberculosis) radiometric system, which was a major breakthrough and was later replaced by Mycobacterium growth indicator tube (MGIT) system for growth (Ardito et al., 2001). 
This also proved to be an excellent technique to perform first- and second-line DST for drugs. A comparison of three techniques for growth, two in liquid and one in solid media, revealed that the semiautomated BACTEC 460 radioactive and fully automated BACTEC 960 could provide reports in 4-12 days, while solid media took 3-4 weeks (Tortoli et al., 1999), this represented a clear breakthrough. These techniques require a minimum of 10-100 M. tuberculosis to grow a culture (Heifets et al., 2000). The sensitivity of these techniques ranges from 80 to $95 \%$, with specificity as high as $98 \%$.( Kenyon et al., 2002)

\section{Limitations}

Smear microscopy and culture are the oldest and most established techniques for TB diagnosis but have some limitations. Sensitivity in microscopy is dependent on the skill of the technician as well as the bacterial load, which typically ranges from $10^{3}$ to $10^{5}$ bacilli per $\mathrm{ml}$ of sputum. False negatives cannot be completely avoided, especially in paucibacillary sample (Odubanjo et al., 2011) In respect to culture, microscopy has low sensitivity, with no ability to distinguish between species of M. tuberculosis (Das et al., 2019).

Though culture is the gold standard for diagnosing tuberculosis, with high sensitivity of 10 bacilli per $\mathrm{ml}$, it also has limitations. The wait of 6-8 weeks for test results causes delays in diagnosis and treatment.(Asmar et al., 2015). The establishment of TB culture labs in peripheral areas, or even in every district is not possible in low-income and developing countries; these techniques require good infrastructure with facilities such as BSL II or BSLIII containment labs with highly trained technical staff( Nienhaus et al., 2011).

\section{Rapid detection of drug resistance}

Although the techniques discussed earlier are capable of TB detection, the sensitivity (in sputum microscopy) or the duration to arrive at diagnosis (in culture) is often less than ideal. Moreover, those techniques cannot address the increase in drug resistant TB. Therefore, development of a method which not only reduces the turnover time but can address drug resistance was a dire need. The rapid techniques followed the molecular changes in the genes targeted by the two most important anti-TB drugs, rifampicin and isoniazid.

Rifampicin inhibits the DNA-dependent RNA polymerase $r p o B$ gene. In almost $96 \%$ of MDR cases of $M$. tuberculosis isolates there are mutations in the $81 \mathrm{bp}$ region coding $507-533$ of the $r p o B$ gene, famously called hot spot region.(Ninan et al., 2016) Meanwhile, for isoniazid resistance that targets both the kat $G$ and inh $A$ genes; the highest level of resistance and the most prevalent mutations are found in the region of S315T of the kat G (Bollela et al., 2016). Whereas in $\operatorname{inh} A$, low levels of resistance to isoniazid is seen at $-15 \mathrm{C} / \mathrm{T}$ (Vilchèze et al., 2014) The detection of mutations for Rif in $r p o B$ serves as a surrogate for MDR resistance (Mani et al., 2001). Many techniques to detect genetic abnormalities have been developed; they including InnoLipa, Rif TB Inno Lipa Mycobacteria V2 and Genotype MTDR plus (Kristen et al., 2019). These techniques have since advanced into more sensitive and faster techniques, like molecular beacon tests and nucleic acid amplification assays.

\section{Probe-based assays}

The assays are rapid and probe-based, involving multiplex PCR with sophisticated reverse hybridization with specific probes tested on nitrocellulose strips. The first few assays that used these techniques are INNO-LiPA®Rif.TB and INNO-LiPA MYCOBACTERIA v2 developed by Innogenetics (Tortoli et al., 2003). More advanced assays, such as GenoType ${ }^{\circledR} M T B D R p l u s$ (Hillemann et al., 2007), are being used to detect both $M$. tuberculosis and resistance to rifampicin and isoniazid. Though these techniques cannot replace the gold standard, they can reduce the time gap between the diagnosis and treatment. Probe-based assays offer sensitivity of up to $92.2 \%$ and specificity of $99.3 \%$. The original Genotype ${ }^{\circledR}$ MTBDRsl assay ver.1 (HainLifescience, Nehren, Germany), is able to detect resistance to second line drugs by detecting mutations in gyr $A$, rrs and embB genes. Whereas, the advance version of the assay (i.e., MTBDRsl v2.0) detects eis promoter region and gyrB mutations in addition to the mutations detected in version 1, which improves the performance of the assay (Lacoma et al., 2012). Then there is advance version of the technique 
incorporated gyrA, gyrB, rrs and eis genes to detect resistance to secondline drugs fluoroquinolones and second line injectables(Tagliani et al., 2015). The sensitivity and specificity (95\% CI) were $100 \%$ and $90 \%$ respectively. According to the WHO's recommendations, the GenoType ${ }^{\circledR} M T B D R$ plus and Genotype ${ }^{\circledR M T B D R s l}$ ver.2 cannot replace the conventional culture methods used to detect MTB but can be used in the screening of MDR and extensively drug resistance $\mathrm{XDR}$ (Migliori et al., 2008).

\section{Limitations}

Probe-based assay are gene-based, and thus cannot differentiate between live and dead bacteria. Though they are fast to give a sensitive result, many countries and the WHO guidelines do not allow their use on a smear-negative sample, which means these methods do not necessarily address the challenge of quick diagnosis and immediate treatment (Chopra et al., 2020). In addition, the sensitivity of this method for extrapulmonary samples is very low. The rate of false positives for resistance cannot be overlooked, as silent mutations cannot be checked. The techniques are not so develop to test all the drugs used as antituberculosis and many drugs must be tested on liquid culture media for sensitivity.

\section{Molecular beacons and nucleic acid amplification-based tests}

The researchers were heading towards faster techniques to diagnose tuberculosis. Molecular beacons are like nucleic acid hybridization probes. These probes, attached to turn-on fluorescent beacons, target a specific region of DNA and bind to the required sequence. The phenomenon is so precise that a single nucleotide change prevents the beacon from binding and fluorescing. These assays allow the detection of mutation in less than $3 \mathrm{~h}$ without a loss of sensitivity and specificity (Nyendak et al., 2009). The specificity and sensitivity of these beacon tests is greater than $97.5 \%$ and $100 \%$, respectively, for rifampicin. Though it cannot replace smear microscopy and culture, nucleic acid amplification assays are currently the most popular diagnostic procedures in multiple settings. The turnover time is around
$2 \mathrm{~h}$, requiring less than 10 bacilli per $\mathrm{ml}$, and offering high specificity and sensitivity.

The Xpert ${ }^{\circledR M T B} /$ RIF (Cephaid) assay, one example of this type, is a powerful tool for tuberculosis control. It identifies portions of the rifampicin resistance determining region (RRDR), part of the $r p o B$ gene in $M$. tuberculosis complex (Mboowa et al., 2014). All five overlapping molecular probes, A to E covers the whole $81 \mathrm{bp}$ core region of the rpoB gene. Some of the techniques based on nucleic acid amplification tests (NAAT) and molecular beacons (Sharma et al., 2018) along with their ability to detect resistance to drugs, are listed in Table 1 (Alemu et al., 2020). Amplified Mycobacterium Tuberculosis Direct Test (Boehme et al., 2010; Boehme et al., 2011; Kenyon et al., 2002; Sollai et al., 2014). The use of Xpert®MTB/RIF (Cephaid) has revolutionized TB treatment and companies are striving to expand the detection limits and sensitivity for a wider panel of drugs. These and other advances are discussed further in the section-New Era of diagnostics.

\section{Limitations}

The technology used in NAAT is again genebased, and whether the detection is of a live or a dead bacterium cannot be differentiated. Techniques such as Xpert®MTB/RIF (Cephaid) are quick and can be used at the point of care, but are expensive, which make it difficult for low income and developing countries to afford. The sensitivity of the technique is low in extrapulmonary samples(Cuevas et al., 2011).

\section{Non-microbiological diagnostic techniques for latent tuberculosis infection}

The diagnosis of TB used to be based on clinical investigation before the rise of technology. The diagnostic techniques and their advances have given a microbiological response to the clinician, thus increasing specificity for case detection and true disease treatment. However, diagnosis of latent TB infection (LTBI) and the test to detect the presence of $M$. tuberculosis in the patient was based on the hypersensitive reactions thus associated. LTBI is defined as a state of persistent immune response to stimulation by $M$. tuberculosis antigens with no clinical evidence of active TB infection (Kasambira et al., 2011). Since, there is no "gold standard" test for LTBI, the burden is 
not known with certainty. However up to onethird of the world's population is estimated to be infected with $M$. tuberculosis (Kenyon et al., 2002; Bokhari et al.,1987) and the vast majority have no signs or symptoms of TB disease. Though they are not infectious, still they are at risk for active TB disease and for becoming infectious (Kasambira et al., 2011). With one-fourth of the global burden of TB, 40 percent of the population of India is infected with MTB. Several studies have shown that, on average $5-10 \%$ of those infected will develop active TB disease over the course of their lives, usually within the first 5 years after initial infection (Pai, et al., 2015). So, in order to achieve End-TB targets within the stipulated time period, it is essential to address this part of the population.

There is no gold standard method for diagnosing LTBI. A tuberculin skin test (TST) and interferon gamma release assay (IGRA) require a competent immune response in order to identify people infected with TB, and these are not perfect tests for measuring progression to active disease.

Current guidelines recommend using only two of the commercially available IGRAs (QuantiFERON®-TB Gold In-Tube and TSPOT®.TB)(Sollai et al., 2014). The Guideline Development Group(GDG) concluded that comparison of TST and IGRA in the same population does not provide strong evidence that one test should be preferred over the other for predicting progression from LTBI to active TB disease(Biraro et al., 2016 )TST may require significantly fewer resources than IGRA and thus may be more practical in resource-constrained settings.

The GDG also noted that equity and access may affect the choice and type of test used. The preferences of clients and programmes are, however, affected by several factors, such as the requirement for sophisticated laboratory infrastructure (for IGRA) and possible additional costs incurred by clients for travelling and by organizations for facilities and testing. The GDG recommended the two tests as equivalent options, with relatively similar advantages and disadvantages. The less accurate performance of these tests can lead to false-negative results, particularly for young children and immunecompromised individuals, such as people living with HIV. Hence, LTBI testing should not be a requirement for initiating TB-preventive treatment for people living with HIV and child household contacts aged less than 5 years, particularly in countries with a high TB incidence, given that the benefits outweigh the risks(Klausner et al., 1993).

The availability and affordability of the tests could determine which LTBI test is used. Other considerations include the structure of the health system, feasibility of implementation and infrastructure requirements. BacilleCalmetteGuérin (BCG) vaccination plays a decisive role in reducing the specificity of TST, suggesting a wider choice of IGRA-only strategies. The GDG noted, however, that the impact of BCG vaccination on the specificity of TST depends on the strain of vaccine used, the age at which the vaccine is given, and the number of doses administered. When BCG is given at birth, as is the case in most parts of the world, it has a variable, limited impact on TST specificity (Zwerling et al., 2011) Therefore, the GDG agreed that a history of BCG vaccination has a limited effect on interpretation of TST results later in life; hence, BCG vaccination should not be a determining factor in selecting a test.

Operational difficulties should be considered in deciding which test to use. IGRAs are more costly and more technically complex to perform than the TST. For example, IGRA requires a phlebotomy, which can be difficult, particularly in very young children, laboratory infrastructure, technical expertise and expensive equipment. However, only a single visit is required to obtain a result, although patients may have to make a second visit to get the result. TST is less costly and can be performed in the field, but it requires a cold chain for storage $\left(2-8^{\circ} \mathrm{C}\right)$ two health care visits, and training in intradermal injection, reading and interpretation.

Blood tests and skin tests are sensitive enough to support the presence of latent TB, but active disease cannot be ruled out-less than half of all cases are likely to be the result of latent TB reactivation. There are many tests to detect interferon gamma in whole blood. Commercially available assays include Quantiferon In-Tube $\mathbb{R}$ (Cellestis Australia), the T.SPOT.TB® (Oxford Immunotech) (Islam et al., 2013) A meta-analysis of different studies compared the specificity of IGRA to TST, finding $85 \%$ to $95 \%$ and $45 \%$ to 
60\% respectively. The WHO has even issued a guideline to stop using serological tests for diagnosis in countries with high TB burdens.

\section{New Era of Diagnostic}

Advances in the diagnosis of TB have been incremental, in step with advances in research and technology. New techniques have been developed, with some approved by the WHO and others by the US FDA.

COBAS TaqMan MTB is a real-time PCR technique developed by Roche Diagnostics and approved by the FDA. The technique detects 16sRNA of $M$. tuberculosis in a smear-positive sample with a turnaround time of $2.5 \mathrm{~h}$ (Yang et al., 2011) Studies have shown that the sensitivity of the assay is $96.9-98 \%$ in smear-positive samples, and $34.9-79.5 \%$ in smear-negative samples. It is an easy technique to implement, one which does not require an advanced laboratory, but its limitations cannot be ignored. Notably, it cannot detect drug resistance, a major consideration for point-of-care testing.

Table 1. Techniques in modern era used for detection of tuberculosis and their ability to detect resistance.

\begin{tabular}{|c|c|c|}
\hline Technology & Amplification reaction & Test detects \\
\hline EasyNAT & Cross priming, Amplification & M. tuberculosis \\
\hline Xpert TB/RIF & RTPCR & Resistance to rifampicin \\
\hline Xpert XDR & RTPCR & $\begin{array}{l}\text { Resistance to fluoroquinolone, } \\
\text { aminoglycosides and isoniazid }\end{array}$ \\
\hline RPA & $\begin{array}{l}\text { Recombinase } \\
\text { amplification }\end{array}$ & M. tuberculosis \\
\hline Truenat & PCR based Semi & Resistance to rifampicin \\
\hline Genedrive & PCR & M. tuberculosis \\
\hline Amplified MTD & $\begin{array}{l}\text { Transcription-mediated, } \\
\text { Amplification }\end{array}$ & M. tuberculosis \\
\hline COBASWTaqMan W MTB Test & RT-PCR & M. tuberculosis \\
\hline $\begin{array}{lr}\text { LoopampW } & \text { Tuberculosis } \\
\text { Complex Detection Reagent Kit }\end{array}$ & LAMP & M. tuberculosis \\
\hline $\begin{array}{l}\text { Genotype MTBDR } \\
\text { Plus \& MTBDRsl }\end{array}$ & $\begin{array}{l}\text { Multiplex PCR and reverse } \\
\text { hybridization }\end{array}$ & $\begin{array}{l}\text { Resistance to rifampicin, } \\
\text { isoniazid, fluoroquinolones and } \\
\text { aminoglycosides }\end{array}$ \\
\hline
\end{tabular}

Loop-mediated isothermal amplification, or LAMP, is a technique developed by Eiken Chemical Co. Ltd. of Japan. LAMP is based on an isothermal nucleic acid amplification technique, which amplifies nucleic acid at a constant temperature without the need for a thermal cycler. This method amplifies even low quantities of DNA with high specificity and a turnaround time of less than $1 \mathrm{~h}$ (Mitarai et al.,2011). Many studies have shown that the technique is simple and easy, though it provides higher sensitivity for smearpositive samples $(92.1 \%-100 \%)$ than for smearnegative samples (52.1\%-90.3\%). Notably, aerosol contamination due mishandling and improper techniques may result in false positive results, and the technique does not detect any markers of drug resistance.
EasyNAT TB is an isothermal nucleic acid amplification technique from Ustar Biotechnologies Co. of China. The technique, which offers a turnaround time of $90 \mathrm{~min}$, has been approved by the FDA. However, EasyNAT does not allow detection of mutations in drug resistance markers.

The gendrive is a technique developed by Epistem (Manchester, UK) for the diagnosis of tuberculosis from samples such as sputum and urine. It is a benchtop test that uses real-time detection by PCR amplification to detect TB with a sensitivity $>90-95 \%$, and specificity $>95 \%$ with the POC of $<45$ minutes (Amplified Mycobacterium Tuberculosis Direct Test Package Insert). Truenat is a technique developed by Molbio Diagnostics Private Ltd, Goa India. The company has developed POC technique which is economical 
semi-automated and quantitative which can detect tuberculosis in the peripheral health centers in developing and low-income countries (Ruth et al., 2015).

True NAAT MTB is a chip-based NAAT technique approved by the WHO. Its turnaround time is $<60 \mathrm{~min}$. This assay can also detect mutations in the $r p o B$ gene to check for resistance to rifampicin.(Ling et al., 2008). This molecular technique give a see through in the shortest time in all the techniques being endorsed in public care. While liquid culture technique remains the gold standard in TB, diagnosing gene sequencing appears to be more sensitive in diagnosing and detecting resistance. The advancement in the field of geneXpert technology developed by Cephaid has been rapid. GeneXpert Omni is a single cartridge based for mobile diagnosing, not evaluated by WHO. XpertMtb Rif/Ultra and Xpert XDR is a next-generation cartridge-based system for the diagnosis of $\mathrm{TB}$, resistance to rifampicin, isoniazid and second-line drugs. The technique was evaluated by the WHO, which issued guidance for its use in 2017. The test has a turnaround time of $<2 \mathrm{~h}$. The Xpert MTB/RIF Ultra amplifies two targets IS6110 and IS1081. It covers 25 different RRDR region of $r p o B$ gene. The Ultra technique has been made more sensitive, reducing the detection limit from 112.6 $\mathrm{cfu} / \mathrm{ml}$ of sputum to $15.6 \mathrm{cfu} / \mathrm{ml}$ (Horne et al., 2019). A study performed by Foundation for Innovative New Diagnostics (FIND) revealed that Ultra was $5 \%$ more sensitive than geneXpert MTB/Rif. The higher sensitivity raises the issue of non-specificity, which is a substantial concern in patients previously treated for TB. However, the assay is still under development and the WHO has not evaluated the test(Horne et al., 2019).

GeneXpert Omni is a new method; it is a single cartridge-based technology which detects tuberculosis and resistance to rifampicin. The machine is battery operative and portable with a shelf life of $4 \mathrm{~h}$.

In 2017, Genomics England, the National Institute for Health Research (NIHR) and the Wellcome Trust worked in partnership to develop whole genome sequencing (WGS)(Stark et al., 2019). WGS has proven to be an accurate diagnosing tool for the detection of TB and drug resistance. It also allows the detection of mutations in the regions other than the regions covered in different diagnostic techniques. The technique provides a quick and complete view of the genotype of $M$. tuberculosis. Many studies reveal that WGS is $99 \%$ in concordance to DST of drugs in culture based medium for drugs rifampicin, isoniazid, pyrazinamide, fluoroquinolones, kanamycin, ethambutol, streptomycin and ethionamide. Despite its benefits, this technique is being used for diagnosis only in developed countries and those with low disease burden. Moreover, the complexity of data interpretation and result assessment makes the technique tough and mandates the requirement of specialist in bioinformatics and medical microbiology.

\section{CONCLUSION}

We have covered the methods to diagnose of tuberculosis, from recognition of symptoms to sputum microscopy, and from culture to nucleic acid amplification tests. The target we have set for ourselves, as sustainable development goals (SDGs) or through END TB, sound unrealistic given the current progress, but advancements in diagnosis will definitely be one of the key components necessary to achieve them.

Accurate, fast diagnosis is the first step to move toward the elimination of TB. Undoubtedly, TB notification has shown an upward trend in the last decade, thanks to newer diagnostic tools with higher sensitivity. Similarly, the number of TB deaths is showing a significant reduction due to early diagnosis and prompt initiation of treatment. Newer diagnostics have improved the overall sensitivity of TB detection in pediatric patients, and in patients with HIV Coinfection or extra-pulmonary disease. The availability of these newer techniques has left serological tests far behind. However, there is still a long way to go because of the rise in the number of drug-resistant cases, especially in developing and poorer nations. It is possible to bridge the gap between disease and drug resistance detection with the new advances made it the field. We must simultaneously address social determinants of health like poverty, malnutrition, overcrowding, alcohol, tobacco and diabetes if we are to achieve our targets. 


\section{REFERENCES}

Alemu, A., Tadesse, M., Seid, G. et al. 2020. Does Xpert ${ }^{\circledR}$ MTB/RIF assay give rifampicin resistance results without identified mutation? Review of cases from Addis Ababa, Ethiopia. BMC Infectious Diseases 20(1): 1-6.

Amplified Mycobacterium Tuberculosis Direct Test. Package Insert. San Diego, CA, USA: Gen-Probe.

Ardito, F., Posteraro, B., Sanguinetti, M., Zanetti, S., \& Fadda, G. 2001. Evaluation of BACTEC Mycobacteria Growth Indicator Tube (MGIT 960) automated system for drug susceptibility testing of Mycobacterium tuberculosis. Journal of Clinical Microbiology 39(12): 4440-4444.

Asmar, S., \& Drancourt, M. 2015. Rapid culture-based diagnosis of pulmonary tuberculosis in developed and developing countries. Frontiers in Microbiology 6: 1184.

Barberis, I., Bragazzi, N. L., Galluzzo, L., \& Martini, M. 2017. The history of tuberculosis: from the first historical records to the isolation of Koch's bacillus. Journal of Preventive Medicine and Hygiene 58(1): E9-E12.

Biraro, I. A., Kimuda, S., Egesa, M., Cose, S., Webb, E. L., Joloba, M., et al. 2016. The use of interferon gamma inducible protein 10 as a potential biomarker in the diagnosis of latent tuberculosis infection in Uganda. PLoS One 11(1): e0146098.

Boehme, C. C., Nabeta, P., Hillemann, D., Nicol, M. P. , et al. 2010. Rapid molecular detection of tuberculosis and rifampin resistance. New England Journal of Medicine 363(11): 10051015.

Boehme, C. C., Nicol, M. P., Nabeta, P., Michael, J. S. , et al. 2011. Feasibility, diagnostic accuracy, and effectiveness of decentralised use of the Xpert MTB/ RIF test for diagnosis of tuberculosis and multidrug resistance: a multicentre implementation study. The Lancet 377(9776): 1495-1505.

Bokhari, S. Y., Ahmad, A., Shaikh, M. Y., Ahmad, I. 1987. A study of tuberculosis contacts. Journal of Pakistan Medical Association 37(2): 48-52.

Bollela, V. R., Namburete, E. I., Feliciano, C. S., et al. 2016. Detection of kat $G$ and $i n h A$ mutations to guide isoniazid and ethionamide use for drug-resistant tuberculosis. The International Journal of Tuberculosis and Lung Disease 20(8): 1099-1104.

Chakravorty, S., Sen, M. K., \& Tyagi, J. S. 2005. Diagnosis of extrapulmonary tuberculosis by smear, culture, and PCR using universal sample processing technology. Journal of Clinical Microbiology 43(9): 4357-4362.

Chopra K. K. \& Singh S. 2020, Newer diagnostic tests for tuberculosis, their utility, and their limitations. Current Medical Research and Practice 10(1): 8-11.

Cuevas, L. E., Al-Sonboli, N., Lawson, L., et al. 2011. LED fluorescence microscopy for the diagnosis of pulmonary tuberculosis: a multi-country cross-sectional evaluation. PLoS Medicine 8(7): e1001057.

Das, P. K., Ganguly, S. B., Mandal, B. 2019. Sputum smear microscopy in tuberculosis: It is still relevant in the era of molecular diagnosis when seen from the public health perspective. Biomedical and Biotechnology Research Journal 3(2): 77-79.

Heifets, L., Linder, T., Sanchez, T., et al. 2000. Two liquid medium systems, mycobacteria growth indicator tube and $\mathrm{MB}$ redox tube, for Mycobacterium tuberculosis isolation from sputum specimens. Journal of Clinical Microbiology 38(3): 1227-1230.

Herzog, B. H. 1998. History of tuberculosis. Respiration 65: 5-15

Hillemann, D., Rüsch-Gerdes, S., \& Richter, E. 2007. Evaluation of the GenoType MTBDRplus assay for rifampin and isoniazid susceptibility testing of Mycobacterium tuberculosis strains and clinical specimens. Journal of Clinical Microbiology 45: 2635-2640.

Horne, D. J., Kohli, M., Zifodya, J. S., et al. 2019. Xpert MTB/RIF and Xpert MTB/RIF Ultra for pulmonary tuberculosis and rifampicin resistance in adults. Cochrane Database of Systematic Reviews 6.

India TB Report. 2019.

Islam, M. R., Khatun, R., Uddin, M. K., Khan, M. S., et al. 2013. Yield of two consecutive sputum specimens for the effective diagnosis of pulmonary tuberculosis. PloS One 8(7), e67678.

Jureen, P., Engstrand, L., Eriksson, S., et al. 2006. Rapid detection of rifampin resistance in Mycobacterium tuberculosis by Pyrosequencing technology. Journal of Clinical Microbiology 44(6): 1925-1929.

Kasambira, T. S., Shah, M., Adrian, P. V., Holshouser, M., et al. 2011. QuantiFERON-TB Gold In-Tube for the detection of Mycobacterium tuberculosis infection in children with household tuberculosis contact. International Journal of Tuberculosis and Lung Disease 15(5): 628-34.

Kenyon, T. A., Creek, T., Laserson, K., et al. 2002. Risk factors for transmission of Mycobacterium tuberculosis from HIV-infected tuberculosis patients, Botswana. International Journal of Tuberculosis and Lung Disease 6(10): 843-50.

Kim, J. H., Kim, Y. J., Ki, C. S., et al. 2011. Evaluation of Cobas TaqMan MTB PCR for detection of Mycobacterium tuberculosis.

Klausner, J. D., Ryder, R. W., Baende, E., et al. 1993. Mycobacterium tuberculosis in household contacts of human immunodeficiency virus type 1-seropositive patients with active pulmonary tuberculosis in Kinshasa, Zaire. Journal of Infectous Diseases 168(1): 106-11.

Kristen, V. D. \& Jason, E. S. 2019. Molecular diagnostics for Mycobacterium tuberculosis infection. Annual Review of Medicine 70(1): 77-90.

Lacoma, A., García-Sierra, N., Prat, C., et al. 2012. GenoType MTBDRsl for molecular detection of second-line-drug and ethambutol resistance in Mycobacterium tuberculosis strains and clinical samples. Journal of Clinical Microbiology 50(1): 30-36.

Ling, D. I., Flores, L. L., Riley, L. W., Pai, M. 2008. Commercial nucleic-acid amplification tests for diagnosis of pulmonary tuberculosis in respiratory specimens: meta-analysis and meta-regression.

Mani, C., Selvakumar, N., Narayanan, S., \& Narayanan, P. R. 2001. Mutations in the $r p o B$ gene of multidrug-resistant Mycobacterium tuberculosis clinical isolates from India. Journal of Clinical Microbiology 39(8): 2987-2990.

Mboowa, G., Namaganda, C., \& Ssengooba, W. 2014. Rifampicin resistance mutations in the $81 \mathrm{bp}$ RRDR of $r p o B$ gene in Mycobacterium tuberculosis clinical isolates using $\mathrm{Xpert}{ }^{\circledR}$ MTB/RIF in Kampala, Uganda: a retrospective study. BMC Infectious Diseases 14: 481.

McNerney, R., Cunningham, J., Hepple, P., Zumla A. 2015. New tuberculosis diagnostics and rollout. International Journal of Infectious Diseases 32: 81-86.

Migliori, G. B., Matteelli, A., Cirillo, D., \& Pai, M. 2008. Diagnosis of multidrug-resistant tuberculosis and extensively drugresistant tuberculosis: Current standards and challenges. The Canadian Journal of Infectious Diseases \& Medical Microbiology 19(2): 169-172.

Mitarai, S., Okumura, M., Toyota, E., et al. 2011. Evaluation of a simple loop-mediated isothermal amplification test kit for the diagnosis of tuberculosis.

Nienhaus, A., Schablon, A., Costa, J. T., et al. 2011. Systematic review of cost and cost-effectiveness of different TBscreening strategies. BMC Health Services Research 11: 247.

Ninan, M. M., Gowri, M., Christopher, D. J., Rupali, P., et al. 2016. The diagnostic utility of line probe assays for multidrug- 
resistant tuberculosis. Pathogens and Global Health 110(4-5): 194-199.

Nyendak, M. R., Lewinsohn, D. A., \& Lewinsohn, D. M. 2009. New diagnostic methods for tuberculosis. Current Opinion in Infectious Diseases 22(2): 174-182.

Odubanjo, M. O. \& Dada-Adegbola, H. O. 2011. The microbiological diagnosis of tuberculosis in a resource limited setting: is acid-fast bacilli microscopy alone sufficient? Annals of Ibadan Postgraduate Medicine 9(1): 24-29.

Pai, M. \& Rodrigues, C. 2015. Management of latent tuberculosis infection: An evidence-based approach. Lung India: Official Organ of Indian Chest Society 32(3): 205-207.

Parsons, L. M., Somoskövi, A., Gutierrez, C., Lee, et al. 2011. Laboratory diagnosis of tuberculosis in resource-poor countries: challenges and opportunities. Clinical Microbiology Reviews 24(2): 314-350.

Reza, L. W., Satyanarayna, S., Enarson, D. A., Kumar, A. M., et al. 2013. LED-fluorescence microscopy for diagnosis of pulmonary tuberculosis under programmatic conditions in India. PloS One 8(10): e 75566.

Sharma N., et al. 2018. Nucleic acid amplification techniques (NAAT) in post genomics era. Acta Scientific Medical Sciences 2(9): 97-104.

Singh, P., Kumar, S. V., Kachhi, R. 2019. Diagnosis of TB: From conventional to modern molecular protocols. Frontiers In Bioscience 11: 38-60

Singhal, R. \& Myneedu, V. P. 2015. Microscopy as a diagnostic tool in pulmonary tuberculosis. International Journal of Mycobacteriology 4(1): 1-6.

Sollai, S., Galli, L., de Martino, M., \& Chiappini, E. 2014. Systematic review and meta-analysis on the utility of Interferon-gamma release assays for the diagnosis of Mycobacterium tuberculosis infection in children: A 2013 update. BMC Infectious Diseases 14(Suppl 1): S6.

Stark, Z., Dolman, L., Manolio, T. A., Ozenberger, B., et al. 2019. Integrating genomics into healthcare: A global responsibility. American Journal of Human Genetics 104(1): 1320.

Tagliani, E., Cabibbe, A. M., Miotto, P, et al. 2015. Diagnostic performance of the new version (v2.0) of GenoType MTBDRsl assay for detection of resistance to fluoroquinolones and second-line injectable drugs: a multicenter study. Journal of Clinical Microbiology 53(9): 2961 2969.

Tortoli, E., Cichero, P., Piersimoni, C., Simonetti, M. T., et al. 1999. Use of BACTEC MGIT 960 for recovery of mycobacteria from clinical specimens: multicenter study. Journal of Clinical Microbiology 37(11): 3578-3582.

Tortoli, Enrico \& Mariottini, Alessandro et al. 2003. Evaluation of INNO-LiPA MYCOBACTERIA v2: Improved reverse hybridization multiple DNA probe assay for mycobacterial identification. Journal of Clinical Microbiology 41: 4418-20.

Van Pinxteren, L. A., Ravn, P., Agger, E. et al. 2000. Diagnosis of tuberculosis based on the two specific antigens ESAT-6 and CFP10. Clinical and Diagnostic Laboratory Immunology 7(2): 155-160.

Vilchèze, C. \& Jacobs, W. R. 2014. Resistance to isoniazid and ethionamide in Mycobacterium tuberculosis: genes, mutations, and causalities. Molecular Genetics of Mycobacteria 2(4): 431453.

World Health Organization (WHO). 2009. Pathways to better diagnostics for tuberculosis: A blueprint for development of TB diagnostics.

Yang, Y. C., Lu, P. L., Huang, S. C., Jenh, Y. S., et al. 2011. Evaluation of the Cobas TaqMan MTB test for direct detection of Mycobacterium tuberculosis complex in respiratory specimens.
Zwerling, A., Behr, M. A., Verma, A., Brewer, T. F., et al. 2011. The BCG World Atlas: a database of global BCG vaccination policies and practices. PLoS Medicine 8(3): e1001012. 